\title{
The XMM-Newton view of the Crab
}

\author{
M. G. F. Kirsch ${ }^{1}$, G. Schönherr ${ }^{1,2}$, E. Kendziorra ${ }^{2}$, M. J. Freyberg ${ }^{3}$, M. Martin ${ }^{2}$, \\ J. Wilms ${ }^{4}$, K. Mukerjee ${ }^{5}$, M. G. Breitfellner ${ }^{1}$, M. J. S. Smith ${ }^{1}$, and R. Staubert ${ }^{2}$ \\ 1 European Space Astronomy Centre (ESAC), ESA, Apartado - PO Box 50727, 28080 Madrid, Spain \\ e-mail: mkirsch@sciops.esa.int \\ 2 Institut für Astronomie und Astrophysik der Universität Tübingen, Abteilung Astronomie, Sand 1, 72076 Tübingen, Germany \\ 3 Max-Planck-Institut für extraterrestrische Physik, Giessenbachstrasse, 85748 Garching, Germany \\ 4 Department of Physics, University of Warwick, CV4 7AL, UK \\ 5 Department of Astronomy and Astrophysics, Tata Institute of Fundamental Research, Colaba, Mumbai-400005, India
}

Received 28 December 2005 / Accepted 15 March 2006

\section{ABSTRACT}

\begin{abstract}
Aims. We discuss the current X-ray view of the Crab Nebula and Pulsar, summarising our analysis of observations of the source with the EPIC-pn camera on board the XMM-Newton observatory. Different modes of EPIC-pn were combined in order to yield a complete scenario of the spectral properties of the Crab resolved in space and time (pulse phase). In addition we give a description of the special EPIC-pn Burst mode and guidance for data reduction in that mode.

Methods. We analysed spectra for the nebula and pulsar separately in the $0.6-12.0 \mathrm{keV}$ energy band. All data were processed with the SAS 6.0.0 XMM-Newton Scientific Analysis System package; models were fitted to the data with XSPEC 11. The high time resolution of EPIC-pn in its Burst mode $(7 \mu \mathrm{s})$ was used for a phase resolved analysis of the pulsar spectrum, after determination of the period with epoch folding techniques. Data from the Small Window mode were processed and corrected for pile-up allowing for spectroscopy simultaneously resolved in space and time.

Results. The spatial variation of the spectrum over the entire region of the Crab shows a gradual spectral softening from the inner pulsar region to the outer nebula region with a variation in photon index, $\Gamma$, from 2.0 to 2.4. Pulse phase resolved spectroscopy of the Crab Pulsar reveals a phase dependent modulation of the photon index in form of a significant hardening of the spectrum in the inter-peak phase from $\Gamma=1.7$ during the pulse peak to $\Gamma=1.5$.
\end{abstract}

Key words. instrumentation: detectors - stars: neutron - stars: pulsars: individual: PSR 0531+21 - X-rays: stars

\section{Introduction}

Since the discovery of the Crab Pulsar in 1968 (Staelin \& Reifenstein 1968), the Crab has been one of the best studied objects in the sky and it remains one of the brightest X-ray sources regularly observed. With an X-ray luminosity of $\sim 5 \times$ $10^{37} \mathrm{erg} \mathrm{s}^{-1}$, the Crab emits a significant fraction of its energy output in the X-ray band (the total luminosity of the Crab is $\left.\sim 2 \times 10^{38} \mathrm{erg} \mathrm{s}^{-1}\right)$. As a standard candle for instrument calibration, it has been repeatedly studied by many astronomy missions. The 33 ms Crab Pulsar has been observed in almost every band of the electromagnetic spectrum. Its pulse profile exhibits a double peaked structure with a phase separation of 0.4 between the first and the second pulse.

Pulse phase resolved X-ray spectroscopy of the Crab was performed for the first time with OSO 8 (Pravdo \& Serlemitsos 1981). Analyses with a finer phase resolution were published from RXTE (Pravdo et al. 1997) and from Beppo-SAX (Massaro et al. 2000). Both measurements showed a significant hardening of the spectrum in the inter-peak region. RXTE and Beppo-SAX both were unable to resolve the Crab spatially. Recent Chandra observations have provided spatially resolved spectroscopy of the Crab (Mori et al. 2004). These observations, however, suffered from pile-up which had to be especially corrected for. Similar correction methods are discussed for the case of the EPIC-pn Small Window mode in Sect. 4.4.
With the European Photon Imaging Camera (EPIC; Strüder et al. 2001; Turner et al. 2001) in its Burst mode, XMM-Newton provides the unique possibility to measure the Crab spectrum without pile-up when combining one dimensional spatial resolution with a high time resolution $(7 \mu \mathrm{s})$, allowing for spectroscopy simultaneously resolved in space and time (phase). In addition, we derived two-dimensionally resolved spectra using bootstrapping techniques and Monte Carlo simulations to correct XMM-Newton EPIC-pn Small Window mode data.

This paper is organised as follows. In Sect. 2 we give a description of our observations and the XMM-Newton instruments, followed by some technical comments on data analysis of Burst mode observations in Sect. 3. Our results from timing and spectral analysis of the Crab are presented in Sect. 4.

\section{Observations performed by XMM-Newton}

XMM-Newton (Jansen et al. 2001) was launched in 1999 December with an Ariane 5 rocket from French Guyana. It operates six instruments in parallel on its $48 \mathrm{~h}$ highly elliptical orbit: three Wolter type 1 telescopes, with 58 nested mirror shells each, focus X-ray photons onto the five X-ray instruments of the EPIC (Strüder et al. 2001; Turner et al. 2001) and the Reflecting Grating Spectrometers (RGS, den Herder et al. 2001). In addition, a $30 \mathrm{~cm}$ Ritchey Chrétien optical telescope, the Optical Monitor, is used for optical observations (OM, Mason et al. 2001). EPIC consists of three cameras: the two EPIC-MOS 
Table 1. Log of observations of the Crab used in this paper.

\begin{tabular}{lrrccc}
\hline \hline OBS ID & Rev. & $\begin{array}{r}\text { Exp. } \\
{[\mathrm{s}]}\end{array}$ & Mode & Filter & $\begin{array}{c}\text { Position } \\
\text { angle [deg] }\end{array}$ \\
\hline 0122330701 & 56 & 166.5 & SW & T & 269.6 \\
0135730701 & 234 & 298.1 & BU & T & 269.3 \\
0153750201 & 411 & 105.1 & BU & M & 267.3 \\
0153750301 & 411 & 181.7 & BU & M & 267.3 \\
0153750501 & 411 & 201.6 & BU & M & 267.3 \\
\hline
\end{tabular}

BU: Burst, SW: Small Window, T: Thick, M: Medium, Exp.: Exposure time, taking also the life time of the different modes into account. * Rotation between the spacecraft $X-Z$-plane and the plane defined by the spacecraft $X$-axis and celestial North.

cameras use Metal-Oxide Semiconductor CCDs as X-ray detectors, while the EPIC-pn camera is equipped with a pnCCD. Both have been especially developed for XMM-Newton (Pfeffermann et al. 1999; Meidinger et al. 1999; Turner et al. 2001).

EPIC provides spatially resolved spectroscopy over a fieldof-view of $30^{\prime}$ with moderate energy resolution. The EPIC cameras can be operated in different observational modes related to different readout procedures. Detailed descriptions of the various readout modes of EPIC-pn and their limitations are given by Kendziorra et al. (1999), Kuster (1999) and Ehle et al. (2003). The EPIC-pn camera, which provides the highest time resolution (Timing mode: $30 \mu \mathrm{s}$, Burst mode: $7 \mu \mathrm{s}$ ) and moderate energy resolution $(E / \mathrm{d} E=10-50)$ in the $0.2-15 \mathrm{keV}$ energy band is the ideal instrument to observe the Crab. In Burst mode the pile-up limit for a point source is 60000 counts s $^{-1}$, corresponding to a maximum flux of $6.3 \mathrm{Crab}$. The special readout of the Burst mode, however, leads to a loss of spatial resolution in the shift direction. Moreover, the lifetime in Burst mode is only $3 \%$, limiting the mode to observations of very bright sources such as the Crab or very bright transients.

The Crab was observed with the EPIC-pn camera in Burst mode during XMM-Newton's revolutions 234 and 411. Observations were also performed in the EPIC-pn Timing and Small Window modes, however the high count rate of the source implies severe pile-up effects which distort the spectrum and require special treatment in the analysis. The Timing mode data have not been used here, since they do not contain additional information compared to the Burst mode data. The Small Window mode data, however, are used despite of the pile-up distortion, as they provide a two-dimensional spatial resolution of the source.

Table 1 summarises the observations of the Crab with EPIC-pn which were included in our analysis, listing operational modes and total exposure times for each individual observation. The exposure times were corrected for data losses due to telemetry constraints and detector deadtime.

\section{Data analysis for EPIC-pn Burst mode}

In the following section we describe the special techniques of data reduction needed for the EPIC-pn Burst mode in order to be able to derive spectra and pulse profiles of the Crab.

The data sets were processed using the XMM-Newton Scientific Analysis System, SAS 6.0.0. Event times were corrected to the solar system barycentre using the SAS tool barycen. Single and double events were considered for the analysis while FLAG $=0$ was chosen to exclude border pixel events, for which the pattern type is uncertain and thus, the total energy is only known with lower precision.

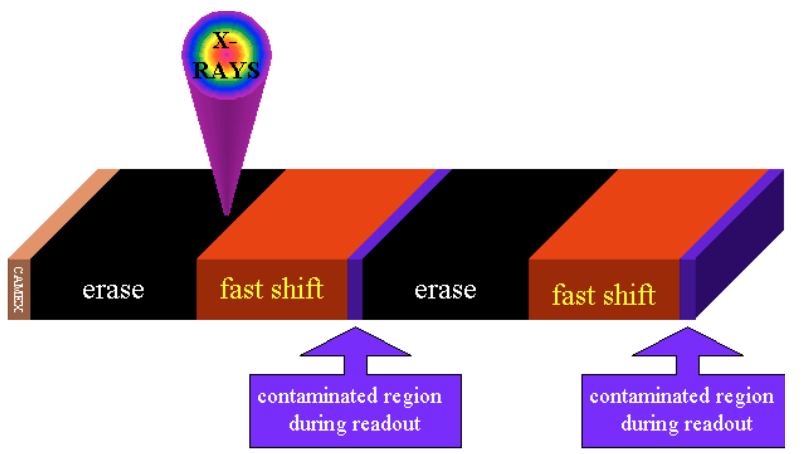

Fig. 1. The special readout in Burst mode, which gives the possibility to observe very bright sources with negligible pile-up.

The Burst mode operates a special readout similar to a tape recorder. Within $14.4 \mu \mathrm{s}, 200$ lines are fast-shifted while accumulating information from the source. Similar to the Timing mode, this leads to the loss of spatial resolution in the shift direction. The stored information is then read out in a standard fashion, ignoring the last 20 lines of the CCD because of contamination by source photons arriving during the readout. The CCD is then erased by a fast shift of 200 lines and immediately afterwards the next Burst readout cycle starts. Figure 1 illustrates this readout procedure. In the following we use SAS terminology for the images, i.e., RAWY represents the CCD line numbers, i.e., the position on the CCD in shift direction from 1-200 and RAWX represents the CCD column numbers, i.e., the position perpendicular to the shift direction from 1-64.

Note that events with RAWY > 180 cannot be used for any kind of data analysis, since they contain severely piled-up data as accumulated from the point-spread function (PSF) wings during the slow readout phase of a Burst cycle. (The HEW of XMMNewton's EPIC-pn PSF is $15^{\prime \prime}$ and one EPIC-pn pixel has a size of $4.128 \times 4.128 \square^{\prime \prime}$.) These data are therefore not transmitted to ground at all. We quantified the effect of pile-up in CCD lines $1 \leq \mathrm{RAWY} \leq 180$ for the measured spectrum of the Crab by extracting spectra with a sliding box of 13 RAWY pixels length, shifted in steps of 10 RAWY pixels. An absorbed power law fixing $N_{\mathrm{H}}$ at $2.76 \times 10^{21} \mathrm{~cm}^{-2}$, as derived from the nebula region of the Crab (Table 2), was fitted to the data. The fit results show that the power law index is only affected for RAWY > 160 while the normalisation seems to be already significantly affected as of RAWY $\sim 140$. Therefore we recommend for general data analysis in the EPIC-pn Burst mode to extract spectra only from RAWY-regions $\leq 160$ for determination of the spectral slope and RAWY $\leq 140$ for determination of the normalisation.

The effects due to the special readout in Burst mode on both the spectral shape and the normalisation of the flux are shown in Fig. 2. For our spectral analysis we excluded events with RAWY $\geq 142$.

The Burst mode in EPIC-pn provides spatial resolution only perpendicular to the CCD readout direction, i.e., in the RAWX direction, which depends on the spacecraft's position angle with respect to the source. To visualise the orientation of the Crab Nebula with respect to the readout direction, we compare in Fig. 3 a Burst mode image of the Crab (revolution 234) to an image obtained in Small Window mode (revolution 56) which was observed under nearly the same position angle (see Table 1). The additional observations in revolution 411 were done with the slight difference of 2.3 in the position angle. This variation, however, is still negligible for the purpose of our analysis. Thus, we can directly compare the two-dimensional image of the Crab 


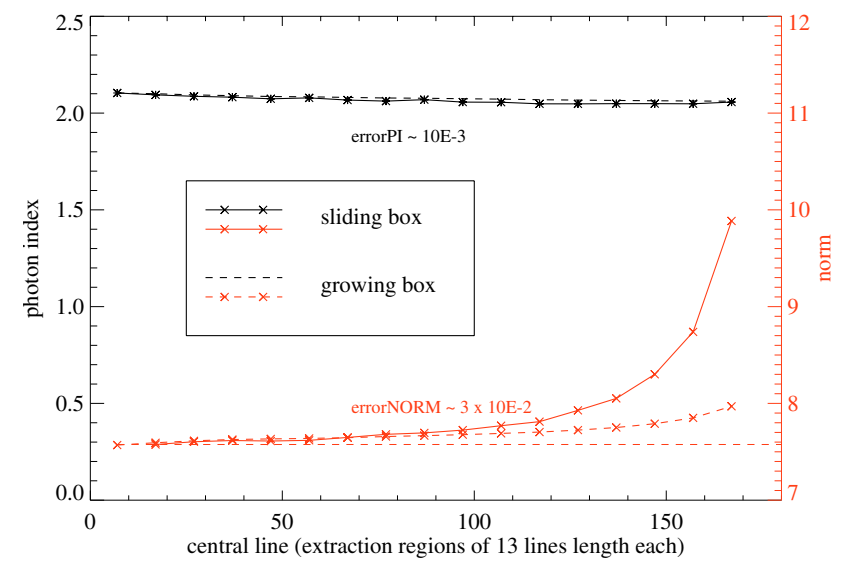

Fig. 2. Variation of spectral parameters with extraction region in EPIC-pn Burst mode. Spectra have been extracted in a sliding box of 13 RAWY pixels in steps of 10 RAWY pixels. An absorbed power law fixing $N_{\mathrm{H}}=2.76 \times 10^{21} \mathrm{~cm}^{-2}$ (see Table 2) derived from the nebula region of the Crab was fitted to the data. In addition we show the variation of the spectral parameters extracting spectra from a growing box in order to visualise the effect as averaged over columns. Spectra should only be extracted for RAWY $\leq 160$ (for determination of the photon index) and RAWY $\leq 140$ (for determination of the normalisation). The background was also taken from a sliding box that contains in first approximation no source photons. Note that the dashed red line without symbols is just to guide the eye with respect to the normalisation parameter variation of the growing box (dashed red one with symbols).

Table 2. Extracted spectra from different regions of interest and spectral parameters.

\begin{tabular}{lcccc}
\hline \hline Spectrum & BGSM $^{*}$ & $\begin{array}{c}N_{\mathrm{H}} \\
10^{21} \mathrm{~cm}^{-2}\end{array}$ & $\Gamma$ & $\chi_{\text {red }}^{2}$ (d.o.f.) \\
\hline total Crab & $\mathrm{C}-\mathrm{B}$ & $2.60(1)$ & $2.046(3)$ & $1.99(3075)$ \\
pulsar & $\mathrm{P}-\mathrm{O}$ & $2.9(3)$ & $1.72(5)$ & $0.91(75)$ \\
nebula & $\mathrm{N}-\mathrm{B}$ & $2.76(4)$ & $2.263(9)$ & $1.24(1394)$ \\
off-pulse & $\mathrm{O}-\mathrm{B}$ & $2.62(4)$ & $2.030(9)$ & $1.23(1296)$ \\
\hline
\end{tabular}

* BGSM: Background Subtraction Method: this column explains which region was used to generate the source and which for the background spectrum (A-B means: A source; B background spectrum.)

C: all Crab (Cols. 22-51), P: pulsar region (Cols. 34-40), N: nebula region (Cols. 22-26), B: background region (Cols. 3-7), O: off-pulse region (Cols. 34-40).

Note that errors are given in terms of $90 \%$ confidence level.

taken in Small Window mode with the one-dimensional cut acquired during Burst mode observations. The extraction regions to derive spectra of the pulsar, nebula and background are also illustrated in Fig. 3.

\section{Data analysis results}

\subsection{Pulse profile and period}

We determined the period of the Crab pulsar using epoch folding techniques. The period $P$ was computed separately for each individual observation in revolutions 234 and 411, using extrapolated values of $\dot{P}$ supplied by the Jodrell Bank Crab Pulsar Monthly Ephemeris (http://www.jb.man.ac.uk). The best pulsar period derived from the data from revolutions 234 and 411 were $33.52130944(2) \mathrm{ms}$ and 33.5341004590(5) $\mathrm{ms}$ for the epochs 51988.6405766415 (MJD) and 52340.6825136183 (MJD) respectively. Thus, the relative deviation of the observed pulse period with respect to the most
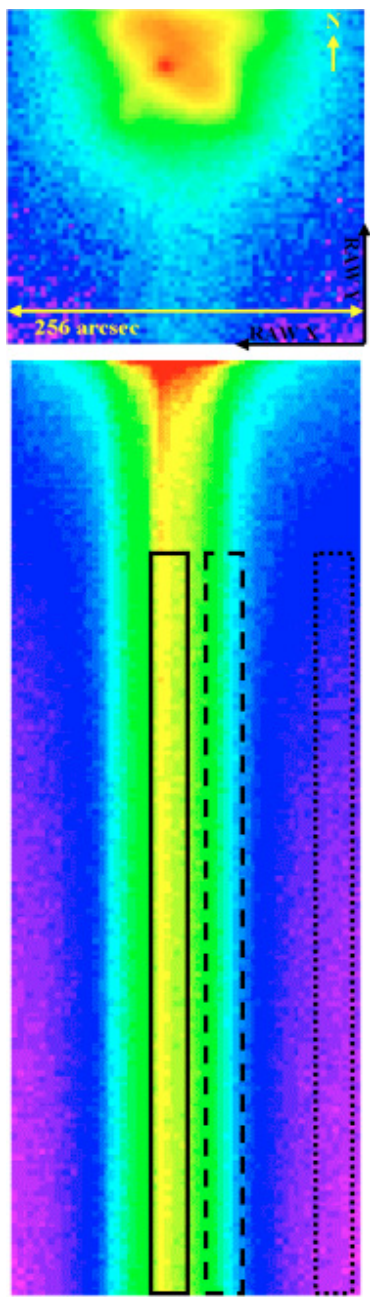

Fig. 3. Upper panel: intensity image of the Crab in EPIC-pn Small Window mode (rev. 56). Lower panel: Crab image in Burst mode. All events in the full energy range $(0.2-15 \mathrm{keV})$ were used. The solid box indicates the pulsar extraction region, while the dashed and dotted boxes are used for nebula and background data extraction respectively.

accurate radio data value available was $\Delta P / P \lesssim 10^{-9}$. Data were combined for detailed analysis from the three observations in revolutions 411 . The folded $0.2-15 \mathrm{keV}$ pulse profile is shown in Fig. 4.

\subsection{Localisation of the pulsar}

As outlined above, spatial resolution in the RAWY-direction is lost when EPIC-pn is operated in Burst mode. To locate the pulsar without use of the attitude in such an image, events in a running window of 5 columns in RAWX-direction were folded with the best-fit period. The pulsed flux for each of those windows was calculated subtracting the off-pulse region (phase 0.568-0.768) as background contribution. The pulsed flux reached its maximum, indicating the location of the pulsar, when the window was centred on Col. 37. In a next step, the pulsar spectrum was extracted taking a larger window of seven columns width (28'. 896$)$ centred at Col. 37 . The spatial variation of the total flux and the pulsed flux is shown in Fig. 5. The peaks of the distributions of the total flux and the pulsed flux are not co-aligned, indicating that the pulsar is not located in the centre of the nebula. The dashed vertical lines show the region chosen for the extraction of the pulsar spectra. 


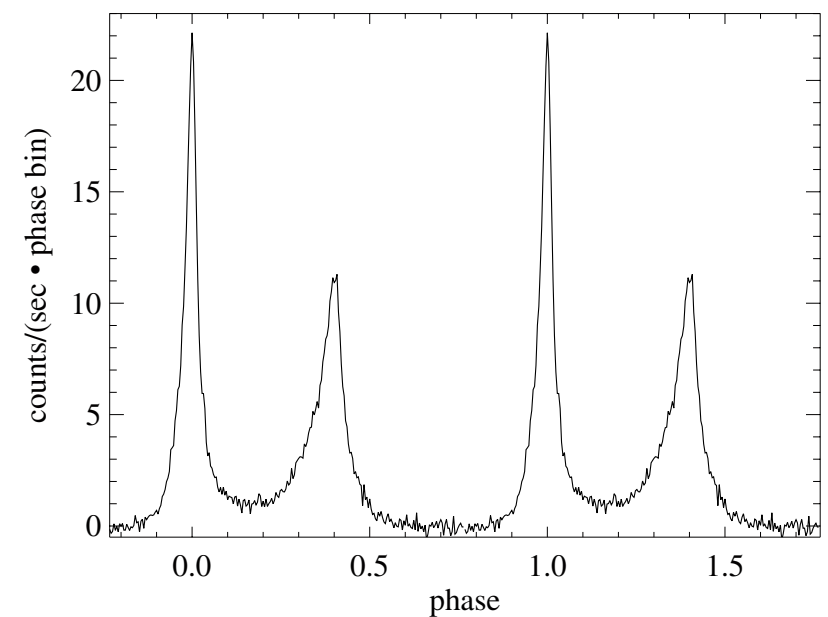

Fig. 4. $0.2-15 \mathrm{keV}$ pulse profile of the Crab Pulsar obtained from the three observations in rev. 411 , using 250 phase bins. The profile is shown twice for clarity.

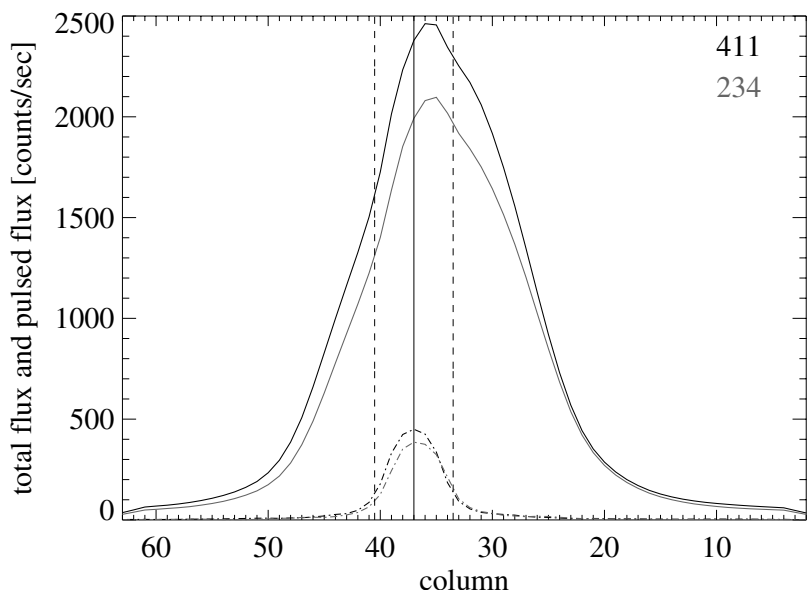

Fig. 5. Variation of the $0.5-15 \mathrm{keV}$ total flux (continuous lines) and the pulsed flux (dashed-dotted lines) over the entire Crab. Note that the flux units are per 7 columns window and renormalised to the same extraction area, using a normalisation factor of $180 / 140$. For the pulsed flux we subtracted the renormalised mean flux from the off-pulse region in phase $0.568-0.768$. The two curves shown are for Rev. 411 and Rev. 234, respectively. The difference in flux is caused by the use of different filters.

\subsection{Spectra of the Crab from EPIC-pn in Burst mode}

Spectra from different regions of interest of the Crab were extracted from the single Burst mode observation in revolution 234 and the three merged observations in revolution 411 , as listed in Table 2. Note that different ways of background subtraction were applied (BGSM). The spectra were fitted with an absorbed single power law model spectrum in XSPEC. The key spectral fit parameters are also listed in Table 2. Fits and model to data ratios are shown in Figs. 6-9.

\subsubsection{Spatial variation of the spectra}

To study the spatial variations of the source in greater depth, spectra were extracted from a running window in RAWXdirection of five columns width. An absorbed power law model was fitted to each spectrum, fixing the equivalent hydrogen column density at $N_{\mathrm{H}}=2.76 \times 10^{21} \mathrm{~cm}^{-2}$ (see Table 2 ) as derived from the nebula region of the Crab. We were able to

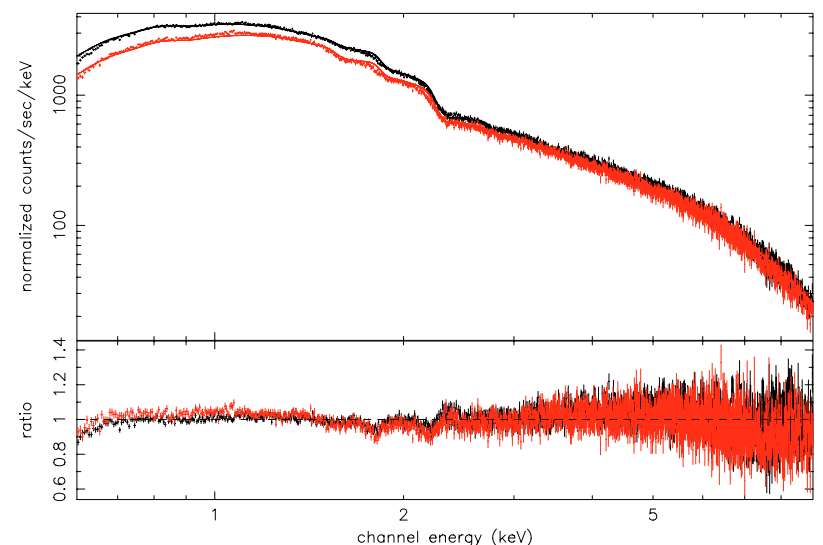

Fig. 6. Spectra of the overall Crab region covering the $0.6-9.0 \mathrm{keV}$ energy band. Data points marked in black and red correspond to revolutions 411 and 234 respectively. The background contributions were subtracted from the data as listed in Table 2.

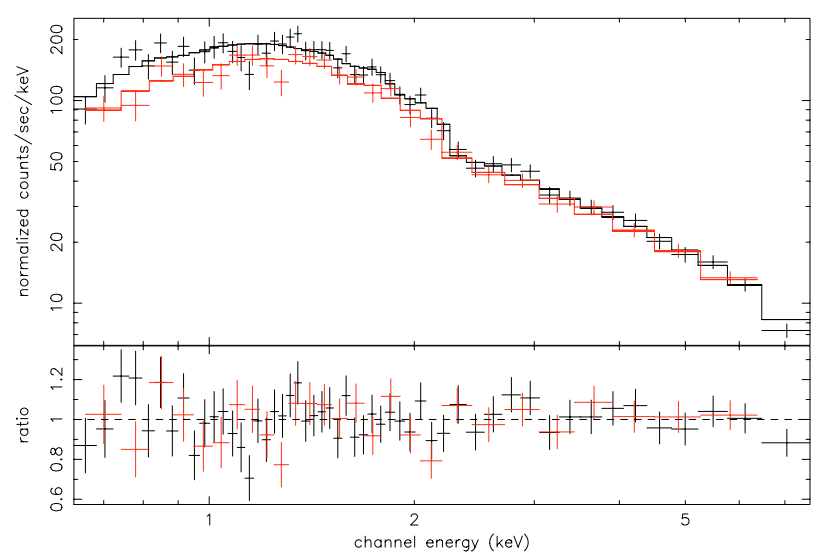

Fig. 7. Spectra of the pulsar region covering the $0.65-8.5 \mathrm{keV}$ energy band. Data points marked in black and red correspond to revolutions 411 and 234, respectively. The background contributions were subtracted from the data as listed in Table 2.

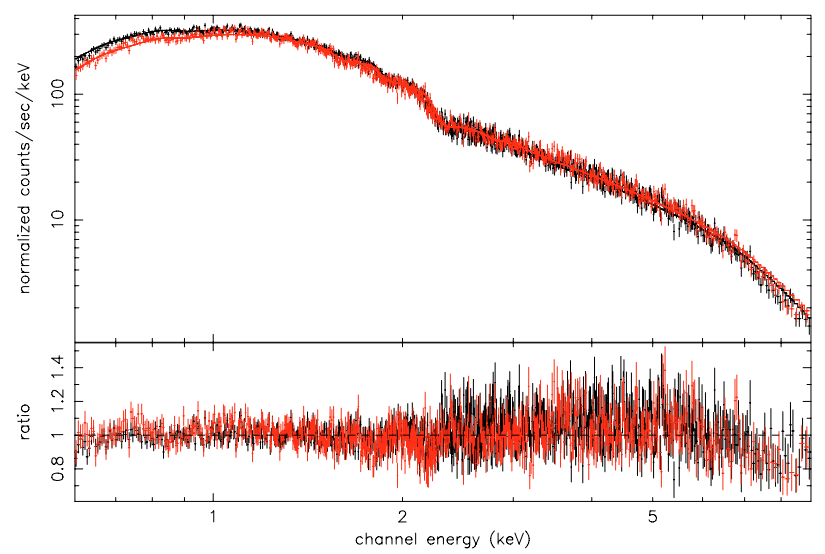

Fig. 8. Spectra of the nebula region covering the $0.6-9.0 \mathrm{keV}$ energy band. Data points marked in black and red correspond to revolutions 411 and 234, respectively. The background contributions were subtracted from the data as listed in Table 2.

distinguish four typical regions as (a) background (Cols. 1-13 and 55-64) (b) outer nebula (Cols. 14-22 and 47-54) (c) inner nebula (Cols. 23-33 and 41-46), and (d) pulsar (Cols. 34-40). The variation of the spectral index along those extraction regions is plotted in Fig. 10. 


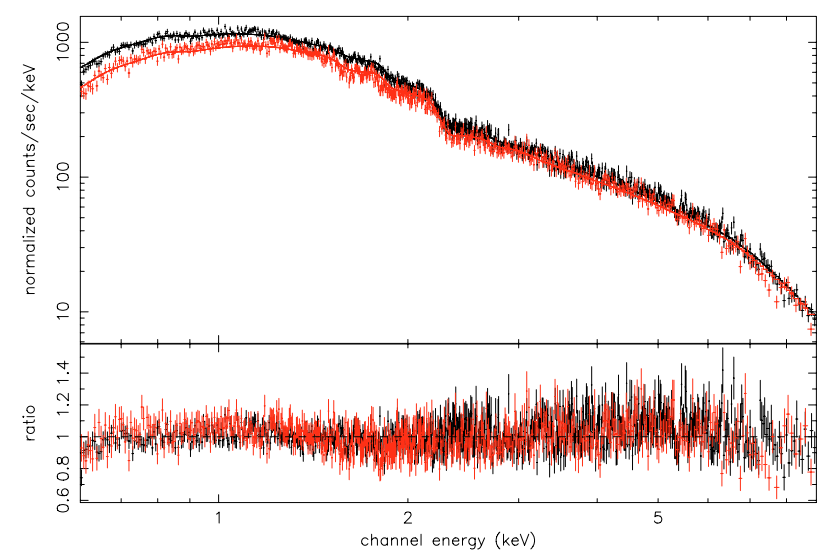

Fig. 9. Spectra of the off-pulse region covering $0.6-9.0 \mathrm{keV}$ energy band. The data points marked in black and red correspond to revolutions 411 and 234, respectively. The background contributions were subtracted from the data as listed in Table 2.

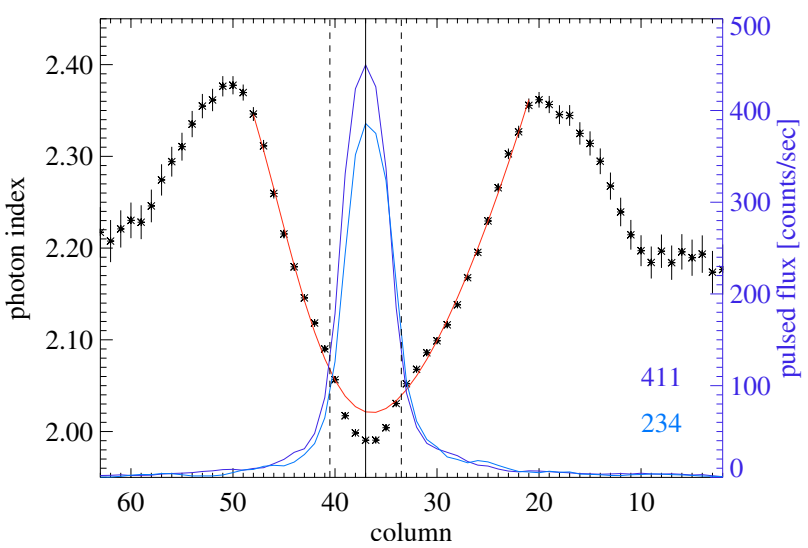

Fig. 10. Spatial variation of the photon index (stars) over the Crab nebula and pulsed flux (solid blue lines) in the energy range $0.6-9.0 \mathrm{keV}$. Note that the flux units are renormalised to the same extraction area by a factor 180/140. For the pulsed flux we subtracted the renormalised mean flux from the off-pulse region (phase 0.568-0.768). Note that since the spectral analysis has been performed without background subtraction the hardening of the spectral index outside the nebula region is influenced by the spectrum of the X-ray background in the Crab direction.

The spectrum is hardest in the region of maximum flux and pulsed fraction (see Figs. 5 and 10). The total variation of the photon index along the Crab is a composed function of the contributions from pulsar and nebula. While all data points outside the pulsar region lie on a parabolic curve, the contribution of the pulsar becomes manifest as an additional dip of the photon index in Cols. 34-40. Note that Fig. 10 is a result of spectral analysis without background subtraction in order to show the hardening of the background outside the nebula region.

\subsubsection{Pulse phase spectroscopy}

Events from the pulsar region (RAWY $<142$, Cols. 34-40) were sorted into 7 pulse phase intervals. For each phase interval we extracted spectra and computed the photon index with $N_{\mathrm{H}}$ fixed at the value derived from the off-pulse region, $N_{\mathrm{H}}=$ $2.62 \times 10^{21} \mathrm{~cm}^{-2}$. The reduced $\chi^{2}$ values are generally acceptable $\left(\chi_{\text {red }}^{2}=1.2-1.3\right)$. For background subtraction, we used the off-pulse region (Cols. 34-40 with phase $0.568-0.768$ ). Plotting the photon index, $\Gamma$, against the phase intervals, we see a modulation of $\Gamma$ with the pulse phase (Fig. 11). The secondary pulse

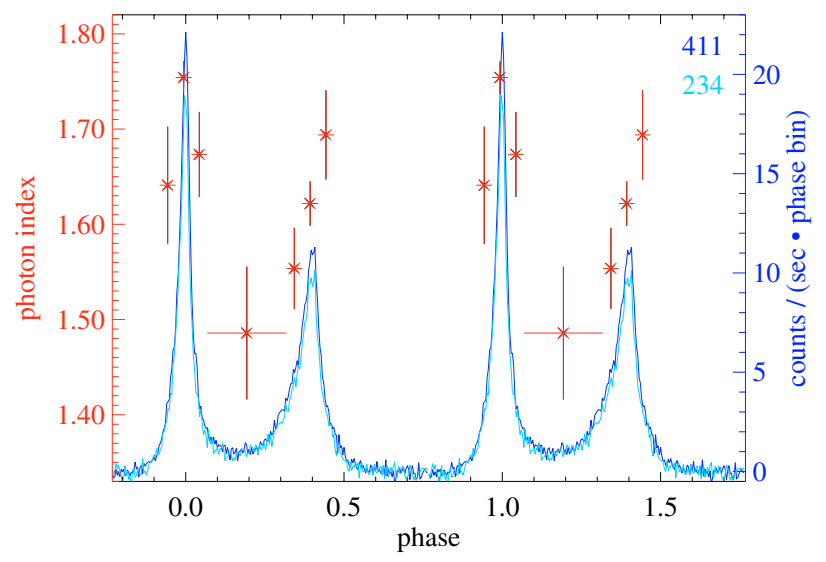

Fig. 11. Spectral variation with pulse phase of the Crab Pulsar. Photon index (red crosses) in the energy range $0.6-6.5 \mathrm{keV}$, pulsed flux: blue lines for the two observations in rev 234 and 411 . For this analysis only data of the Burst mode observations have been used. The flux has been renormalised to the same extraction region by multiplying with a correction factor of 180/140. For the pulsed flux we subtracted the renormalised mean flux from the off-pulse region with phase $0.568-0.768$ and took into account that we measure only $77 \%$ of the encirceled energy in the extraction region of seven columns.

shows a harder spectrum than the main pulse. In the intermediate pulse region the spectrum is found to be harder than in the peak of the pulses. The spectrum of the intermediate pulse region is harder than the spectrum of the main pulse by 0.3 . The spectrum of the second pulse is harder than the spectrum of the main pulse by 0.1 .

The analysis of Massaro et al. (2000) did show, that a two component emission model can account for the variation of the pulse profile with energy and the behaviour of the spectral index with phase. However, no complete model for the X-ray emission from the Crab Pulsar was provided.

\subsection{Spatially resolved spectroscopy of the Small Window mode data}

In Small Window mode, one EPIC-pn CCD is operated in a window of $64 \times 64$ pixels (Fig. 3). Events are read out after an integration time of $3.9812 \mathrm{~ms}$. Due to the high source intensity, observational data of the Crab taken in Small Window mode is expected to be severely affected by pile-up (the limiting count rate in Small Window mode before the onset of pile-up is of the order of 130 counts s $^{-1}$ for a point source, Ehle et al. 2003). However, as outlined above, the Small Window mode provides additional spatial information compared to the Burst mode. In analogy to the analysis of Burst mode data (Sects. 3, 4.3), we extracted Small Window mode spectra of the Crab (single and double events, FLAG $=0$ ) and fitted an absorbed power law $X S P E C$ model to the data. The results of our analysis are visualised in a colour coded diagram in Fig. 12. The variation of the photon index over the $\mathrm{Crab}$ is indicated by the colours of the boxes, representing extraction regions of $153.363 \square^{\prime \prime}$ each. The observed count rates are overplotted as contours. Note that these results are of qualitative nature only and yet have to be corrected for pile-up effects.

In order to estimate the level of data contamination due to pile-up we took two different approaches:

1. By comparing predicted to measured CCD pattern fractions using the SAS task epatplot (Fig. 13). The change in pattern ratios is a sensible indicator of the onset of pattern pile-up 


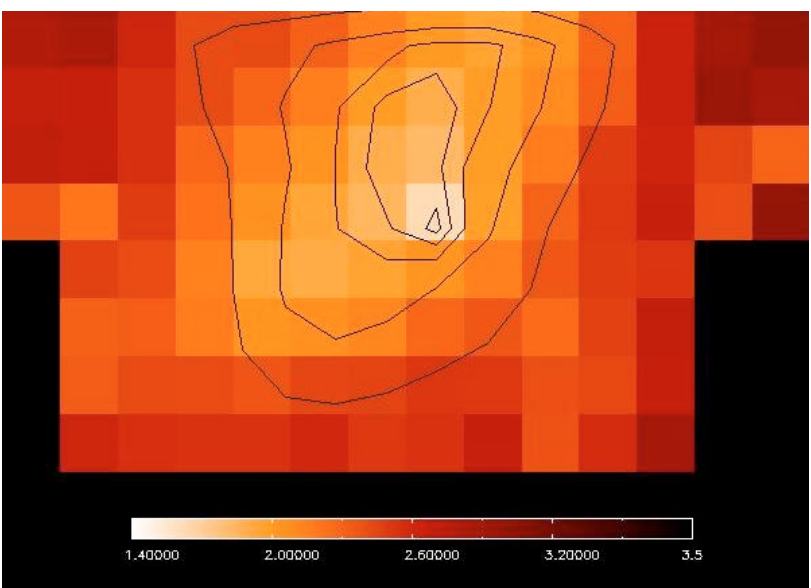

Fig. 12. Two dimensional spectroscopy of the Small Window mode data without correction for pile-up. One coloured box represents a region of 9 EPIC-pn pixels (153.363 $\left.\square^{\prime \prime}\right)$, the colour represents the spectral index (see colour code scale). The count rate contours in Small Window mode are overplotted.

(Two single photons hitting the detector in the same readout frame in adjacent pixels are misinterpreted as a single event of double pattern). Since the lower threshold of the EPICpn camera was $80 \mathrm{ADU}(400 \mathrm{eV})$ higher than the default value for the Small Window mode observation we had to recalibrate epatplot using pattern fractions of non piled-up sources in Small Window mode. This special calibration will be provided to the public in the near future via the standard SAS.

2. Analysing the loss of events due to pile-up by determining the count rate of single columns in Burst mode and comparing them with the corresponding columns in Small Window mode (Fig. 14). Note that for this analysis only events with RAWY $\leq 11$ have been used for Burst mode in order to avoid any effect of pile-up during readout hampering the normalisation (Fig. 2). Figure 14 shows the ratio of the count rates in Burst and Small Window mode, where numbers diverging from 1.0 indicate pile-up.

Both methods confirm that the complete source area of the Crab observation in Small Window mode may only be used for analysis when correcting the data for pile-up. Pile-up effects change both flux and spectral shape of the source data, as with increasing count rate the probability increases that several low-energy events are wrongly detected as single high-energy photons.

In order to quantify the effects of pile-up on the spectral shape, Burst mode data and the Small Window mode data have been compared in one spatial dimension, considering spectra extracted from single CCD columns. This approach simulates for the Small Window mode the loss of spatial resolution along the shift direction in Burst mode. A direct comparison can thus be made for non piled-up data from Burst mode and piled-up data from Small Window mode. Figure 15 shows at the left the resulting average photon indices as a function of count rate (column) for both Small Window and Burst mode data. The piled-up Small Window mode spectra are flatter than the non piled-up Burst mode spectra. This comparison gives a first estimate of the order of pile-up corrections required in general for Small Window mode spectra of Crab-like sources, and will be used in the following to test our theoretical pile-up corrections (Fig. 15, right).

This observational estimate, however, can not be used for a straightforward correction of the two-dimensional spectral
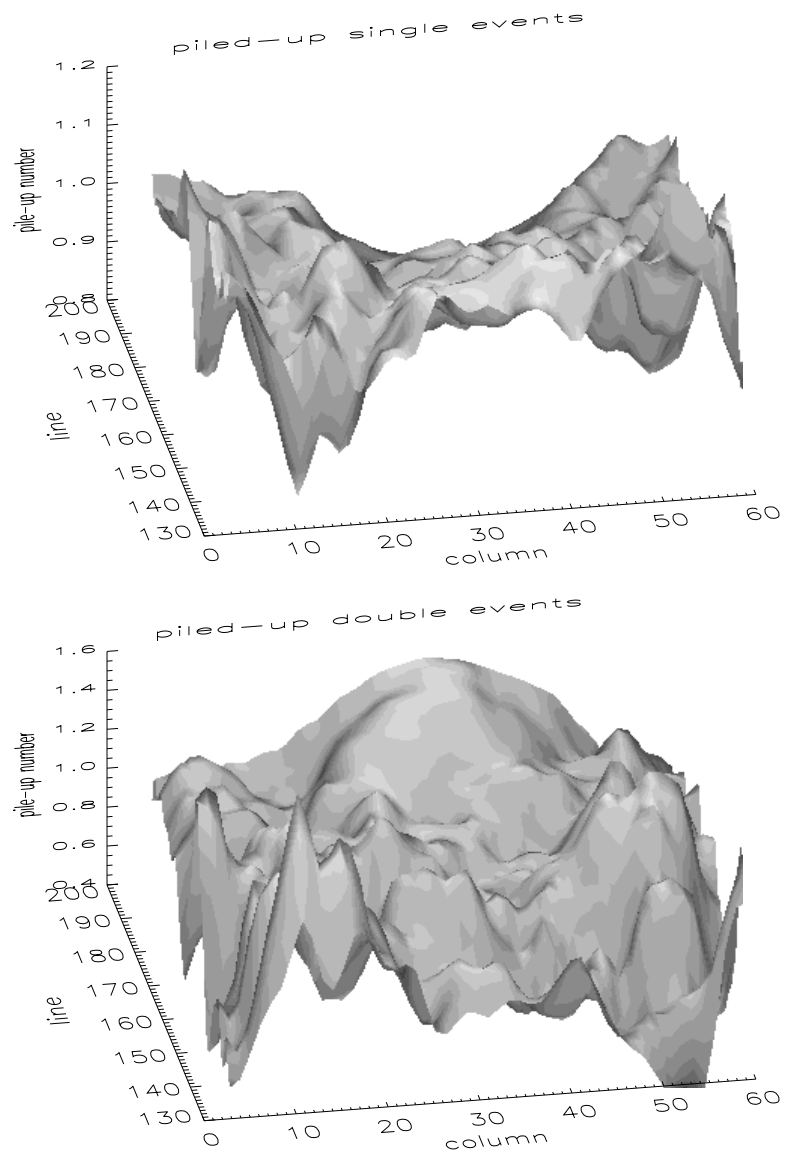

Fig. 13. Pile-up assessment of the Crab observations in Small Window mode. We compared the predicted with the measured pattern fractions using the SAS-task epatplot. epatplot was operated on a grid of event regions with $7 \times 7$ pixels $(834.979 \square$ "). The upper panel shows the deficit in single events, the lower the increase of double events. Both is an indication for pile-up.

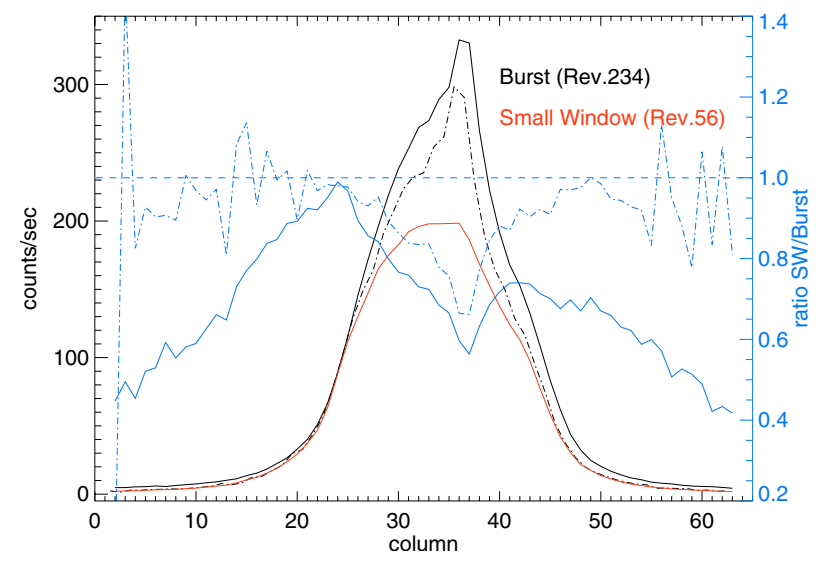

Fig. 14. Count rates and ratio of the count rates in Burst and Small Window mode, where numbers diverging from one indicate pile-up (blue). Black solid: Burst mode count rate, red solid: Small Window mode count rate, black dashed: Burst mode count rate corrected a) for pointing difference Burst and Small Window observation of 0.5 pixels between; b) the extraction region has been reduced to RAWY values from 2-11; c) the lower threshold settings of Small Window mode have been also applied to the Burst mode data. Blue solid: uncorrected ratio of Burst and Small Window mode count rate, blue dashed: corrected ratio of Burst and Small Window mode count rate.

analysis, where the observed count rate assumes values up to 1 count $/\left(s \square^{\prime \prime}\right)$ in the central region of the Crab. In a more 

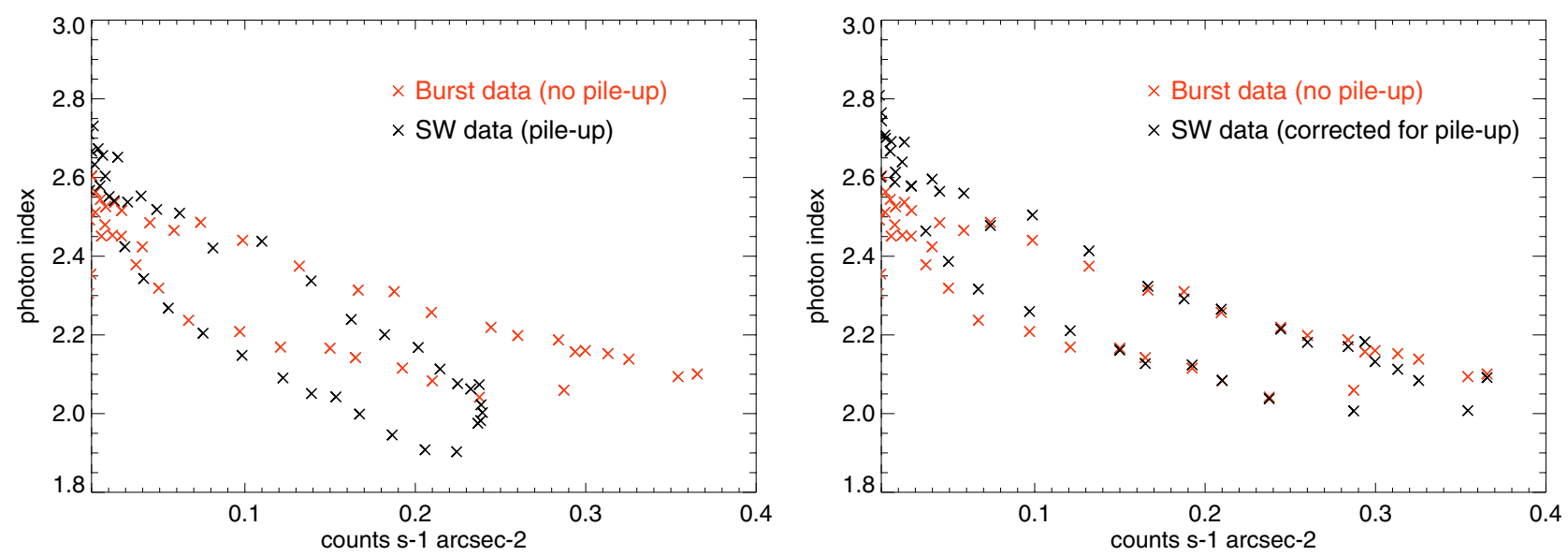

Fig. 15. Left: photon indices as a function of count rate for both Small Window and Burst mode. The piled up Small Window mode spectra show lower photon indices than the non piled-up Burst mode spectra for the same count rates. Right: same data set with pile-up corrections for Small Window data. The Small Window count rates are replaced by the correct values from Burst mode, the photon indices are corrected according to the function $f(r)$ given by (1). Note that the general trend of the data points (lower photon index with higher count rate) reflects the spectral behaviour of the Crab as already shown in Fig. 10.

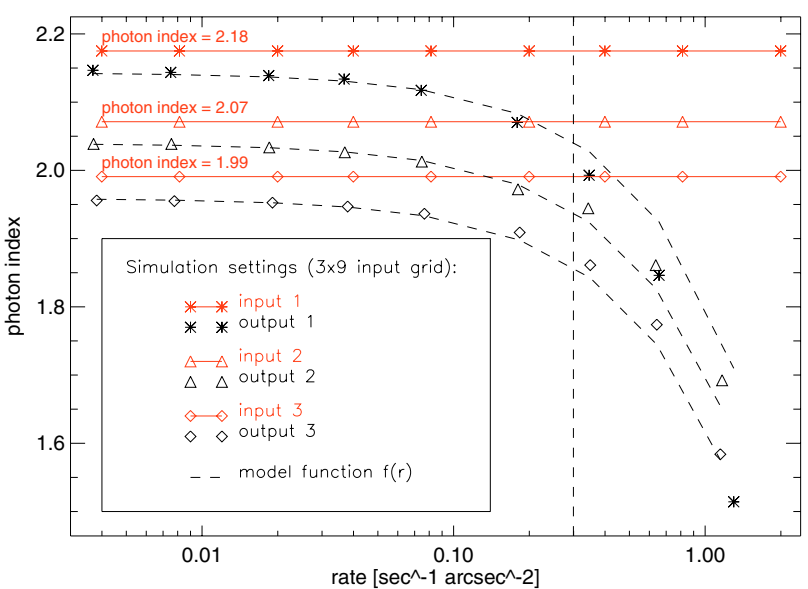

Fig. 16. Result of Monte Carlo simulations to estimate the effect of pileup to the measured spectra depending on photon index and count rate.

general approach, we performed Monte Carlo simulations, implementing the pile-up behaviour of the EPIC-pn CCDs. Using non-contaminated Burst mode spectra to fix the input spectral shape characterised by various photon indices, and varying the input flux, we simulated the effect of the Small Window mode on the source spectra. Comparing the results from spectral fits of Burst input and simulated Small Window output spectra, we derived a correction function $f$ for the photon index with respect to the observed count rate. With the onset of pile-up in Small Window mode, the photon index [p.i. $\left.{ }^{\mathrm{SW}}(r)\right]$ decreases linearly with the observed count rate $r$ :

$f(r)=\left[\right.$ p.i. $\left.^{\mathrm{SW}}(r)\right] \sim\left[\right.$ p.i. $\left.^{\text {BURST }}\right]-$ const. $-\frac{1}{3} \frac{r}{s \square^{\prime \prime}}$.

The negative constant shift (const. $\sim 0.03$ ) of the observed with respect to the true photon index is caused by the unusually high lower threshold of this Small Window mode observation, which leads to changes in the fraction of double and single patterns at low energies and thus to changes of the spectral shape in the low energy regime.

Figure 16 shows the result of our simulations and indicates the shape of the correction function for different initial settings.

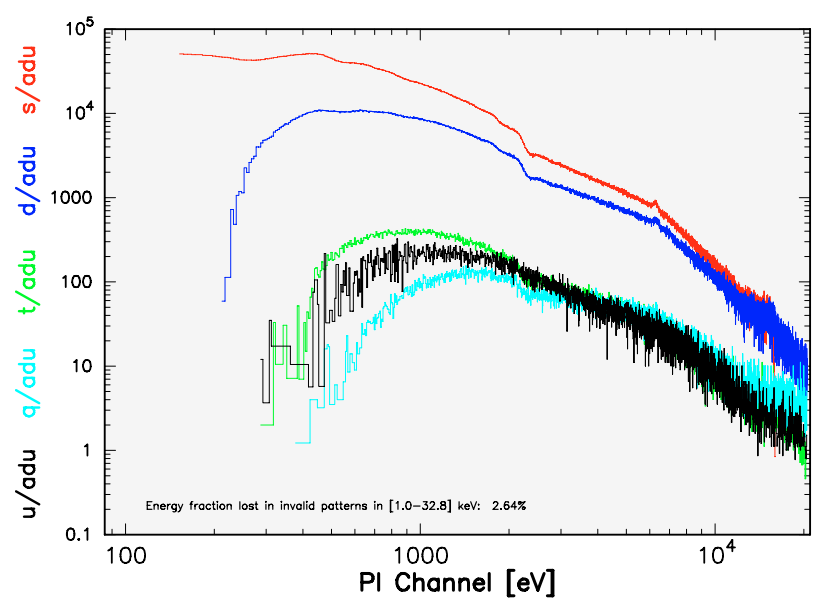

Fig. 17. Distribution of patterns for a merged EPIC-pn Small Window mode observation of $620 \mathrm{ks}$ public data. Internal background has been subtracted using 125 ks merged Closed-Filter observations. Red: singles, blue: doubles, green: triples, light blue: quadruples, black: invalid patterns.

The corrections for the photon index have been applied to the Small Window mode data. The derived correction function (1) is in very good accordance to our independent observational quantification of pile-up effects for the one-dimensionally averaged Small Window data (Fig. 15, right). Correction of the two dimensional spectroscopy of the Crab yields a final photon index of $\Gamma=1.96$ (without correction: $\Gamma=1.6$ ) for the innermost extraction region around the Crab Pulsar.

Note that for that analysis triple and quadruple events can be neglected in the case of the EPIC-pn camera since they only account for less than $4 \%$ of all events, see also Fig. 17.

\section{Conclusion}

Our work provides phase resolved spectroscopy of the modulated flux of the Crab Pulsar in combination with spatially dependent spectral results of the pulsar and the nebula. We measure a temporal hardening of the spectral index in the inter peak region by 0.4 . This confirms the results achieved by Beppo-SAX (Massaro et al. 2000) and RXTE (Pravdo et al. 1997) 
measurements. In addition, we give some insight into the spatial behaviour of the nebula both from one-dimensional EPIC-pn Burst mode and two-dimensional EPIC-pn Small Window mode data. We show that the pulsar region of the Crab Nebula has by far the hardest spectrum getting softer at larger distance from the pulsar. These results are in line with the analysis of XMMNewton EPIC-MOS data (Willingale et al. 2001) and Chandra (Mori et al. 2004).

For the first time we can verify a simulated pile-up correction for XMM-Newton imaging mode data with real data from the one dimensionally resolved Burst mode.

We like to stress that this paper describes in detail how $X M M-N e w t o n$ EPIC-pn data from its special Burst mode need to be analysed addressing both the excellent prospectives and the strength of that mode, but also the caveats that are crucial for an adequate treatment of the data and their exploitation.

Acknowledgements. The XMM-Newton project is an ESA Science Mission with instruments and contributions directly funded by ESA Member States and the USA (NASA). The German contribution of the XMM-Newton project is supported by the Bundesministerium für Bildung und Forschung/Deutsches Zentrum für Luft- und Raumfahrt. The UK involvement is funded by the Particle Physics and Astronomy Research Council (PPARC).

\section{References}

Ehle, M., et al. 2003, XMM-Newton Users Handbook, Issue 2.1 den Herder, J. W., Brinkman, A. C., Kahn, S. M., et al. 2001, A\&A, 365, L7 Jansen, F., Lumb, D., Altieri, B., et al. 2001, A\&A, 365, L1

Kendziorra, E., Colli, M., Kuster, M., et al. 1999, EUV, X-Ray, and Gamma-Ray Instrumentation for Astronomy X, ed. O. Siegmund, \& K. A. Flanagan, Proc. SPIE, 3765, 204

Kuster, M., Benlloch, S., Kendziorra, E., \& Briel, U.G. 1999, EUV, X-Ray, and Gamma-Ray Instrumentation for Astronomy X, ed. O. Siegmund, \& K. A. Flanagan, Proc. SPIE, 3765, 673

Meidinger, N., et al. 1999, EUV, X-Ray, and Gamma-Ray Instrumentation for Astronomy X, ed. O. Siegmund, \& K. A. Flanagan, Proc. SPIE, 3765, 192

Mason, K. O., Breeveld, A., Much, R., et al. 2001, A\&A, 365, L36

Massaro, E., Cusumano, G., Litterio, M., \& Mineo, T. 2000, A\&A, 361, 695

Mori, K., Burrows, D. N., Hester, J. J., et al. 2004, ApJ, 609, 186

Pfeffermann, E., et al. 1999, EUV, X-Ray, and Gamma-Ray Instrumentation for

Astronomy X, ed. O. Siegmund, \& K. A. Flanagan, Proc. SPIE, 3765, 184

Pravdo, S. H., \& Serlemitsos, P. J. 1981, ApJ, 246, 484

Pravdo, S. H., Angelini, L., \& Harding, A. K. 1997, ApJ, 491, 808

Staelin, D. M., \& Reifenstein, E. C. 1968, Science, 162, 1481

Strüder, L., Briel, U., Dennerl, K., et al. 2001, A\&A, 365, L18

Turner, M. J. L., Abbey, A., Arnaud, M., et al. 2001, A\&A, 365, L27

Willingale, R., Aschenbach, B., Griffiths, R. G., et al. 2001, A\&A, 365, L212 